\title{
A renormalisation-group approach to two-body scattering with long-range forces
}

\author{
Thomas Barford and Michael C. Birse \\ Department of Physics and Astronomy, University of Manchester, Manchester, M13 9PL, U.K.
}

\begin{abstract}
We apply the renormalisation-group to two-body scattering by a combination of known long-range and unknown short-range forces. A crucial feature is that the low-energy effective theory is regulated by applying a cut-off in the basis of distorted waves for the long range potential. We illustrate the method by applying it to scattering in the presence of a repulsive $1 / r^{2}$ potential. We find a trivial fixed point, describing systems with weak short-range interactions, and a unstable fixed point. The expansion around the latter corresponds to a distorted-wave effective-range expansion.
\end{abstract}

\section{INTRODUCTION}

Effective field theories (EFT's) offer the promise of a systematic treatment of fewnucleon systems at low-energies. (For a review, see: [1].) They are based on the existence of a separation of scales between those of the low-energy physics: momenta, energies, $m_{\pi}$ (generically $Q$ ), and those of the underlying physics: $m_{\rho}, M_{N}, 4 \pi f_{\pi}$ (generically $\Lambda_{0}$ ). This makes it possible to systematically expand both the theory (defined by a Lagrangian or a potential) and physical observables in powers of $Q / \Lambda_{0}$. Such an expansion will be useful provided it converges rapidly enough.

For weakly interacting systems (such as chiral perturbation theory in the zero- or one-nucleon sectors) the terms in the expansion can be organised according to naive (Weinberg) power counting. The order of a term $\left(Q / \Lambda_{0}\right)^{d}$ in the theory is just $d[2,3]$.

In contrast for strongly interacting systems (such as $s$-wave nucleon-nucleon scattering) there can be new low-energy scales generated by nonperturbative dynamics. In such cases we need to resum certain terms in the theory and this leads to a new power counting $[4,5,6,7,8]$, often referred to as KSW power counting. The theoretical tool which allows us to determine the power counting in these cases is the renormalisation group (RG).

\section{RENORMALISATION GROUP}

The RG is an extension of simple dimensional analysis which has been used to study the scaling behaviour of systems in a wide range of areas of physics. In our work we use a Wilsonian version of the RG [9]. This has the following ingredients:

- Impose a momentum cut-off, $|\mathbf{k}|<\Lambda$ on the low-energy effective theory. 
- Demand that observables be independent of the cut-off $\Lambda$. This corresponds to integrating out physics on momentum scales above $\Lambda$.

- Rescale the theory, expressing all dimensioned quantities in units of $\Lambda$. Before doing this, we need to have identified all the important low-energy scales in our system.

We can then follow the flow of the various coupling constants of the theory as $\Lambda \rightarrow 0$. If there is a clear separation of scales, then the theory should flow towards a fixed point. This is because, for $\Lambda<<\Lambda_{0}$, the only scale left is $\Lambda$ and so the rescaled theory becomes independent of scale.

Near a fixed point, perturbations scale as powers of the cut-off, $\Lambda^{v}$, and these define the power counting for the corresponding terms in the theory: $d=v-1$. The sign of $v$ can be used to classify these terms:

- $v>0$ : irrelevant perturbation, flows towards the fixed point as $\Lambda \rightarrow 0$,

$\cdot v=0$ : marginal perturbation (or "renormalisable" in field-theory terminology), leads to logarithmic flow with $\Lambda$,

- $v<0$ : relevant perturbation, flows away from the fixed point as $\Lambda \rightarrow 0$, making the point an unstable one.

The application of these ideas to two-body scattering by short-range forces can be found in Ref. [8]. Two fixed points were found: a trivial one describing a system with weak scattering, and a nontrivial one describing a system with a bound state at zero energy. The expansion around the nontrivial one is organised by KSW power counting. It is in fact just the effective-range expansion [10], reinvented in modern languange as an EFT.

\section{DISTORTED-WAVE THEORY}

Scattering by short-range interactions in the presence of a known long-range potential $V_{L}$ can be treated by distorted-wave theory. We write the full scattering matrix as

$$
T=T_{L}+\left(1+V_{L} G_{L}\right) \tilde{T}_{S}\left(1+G_{L} V_{L}\right),
$$

where $T_{L}$ is the scattering matrix for $V_{L}$ alone and $G_{L}$ is the corresponding Green's function. The operator $\tilde{T}_{S}$ describes the scattering between distorted waves of $V_{L}$. In terms of a short-range potential $V_{S}$, it satisfies the Lippmann-Schwinger equation

$$
\tilde{T}_{S}=V_{S}+V_{S} G_{L} \tilde{T}_{S}
$$

We regulate this equation by cutting off $G_{L}$ in the basis of distorted waves [11, 12],

$$
G_{L}=M \int^{\Lambda} \frac{d^{3} \mathbf{q}}{(2 \pi)^{3}} \frac{\left|\psi_{L}(q)\right\rangle\left\langle\psi_{L}(q)\right|}{p^{2}-q^{2}+i \varepsilon} \text { (+bound states). }
$$

(Here $p=\sqrt{M E}$ denotes the on-shell relative momentum for two particles of mass $M$.) The use of this basis is crucial for the identification of the scaling behaviour. 
Since the long-range potentials of interest are generally singular as $r \rightarrow 0$, we cannot represent the short-range interaction by a simple $\delta$-function at the origin. Instead we choose a $\delta$-shell form,

$$
V_{S}=V(p, \Lambda) \frac{\delta(r-R)}{4 \pi R^{2}}
$$

Note that we have allowed the potential to be energy $(p)$ dependent. However we have not included momentum dependence since, for the pure short-range-case, this was found to affect only the off-shell behaviour of scattering amplitudes [8].

We require that $V(p, \Lambda)$ vary with $\Lambda$ to keep the fully off-shell DW scattering matrix $\tilde{T}_{S}$ independent of cut-off. Rescaling the resulting differential equation gives us a DW version of the RG equation [12].

\section{REPULSIVE INVERSE-SQUARE POTENTIAL}

To illustrate these ideas we apply them here to a specific example: a repulsive $1 / r^{2}$ potential,

$$
V_{L}=\frac{\beta}{r^{2}}
$$

This potential is analogous to the interaction in a three-body system such as quartet nd scattering, in the limit of an infinite two-body scattering length [13]. It is scale independent and so should be treated as part of a fixed point (and resummed to all orders). It acts in $s$-waves like a centrifugal barrier with "angular momentum"

$$
\lambda=\sqrt{\beta M+\frac{1}{4}}-\frac{1}{2} .
$$

The corresponding DW's are Bessel functions $j_{\lambda}(k r)$ of noninteger order.

The requirement that $\tilde{T}_{S}$ be independent of cut-off leads to a differential equation for $V(p, \Lambda)$,

$$
\frac{\partial V}{\partial \Lambda}=-\frac{M V^{2}}{2 \pi^{2}} \frac{\Lambda^{2} j_{\lambda}(\Lambda r)^{2}}{p^{2}-\Lambda^{2}}
$$

Provided $R<<\Lambda^{-1}$ the DW factors scale as powers of $\Lambda: j_{\lambda}(\Lambda r) \propto(\Lambda R)^{\lambda}$. We can then rescale, defining a dimensionless potential

$$
\hat{V} \propto M R^{2 \lambda} \Lambda^{2 \lambda+1} V
$$

and on-shell momentum $\hat{p}=p / \Lambda$, to rewrite Eq. (7) as an RG equation for $\hat{V}$.

We have found two-fixed points of this equation:

- The trivial one, $\hat{V}=0$. Perturbations around this are of the form $\Lambda^{2 n+2 \lambda+1} \hat{p}^{2 n}$ and can be assigned an order $d=2(n+\lambda)$.

- A nontrivial one. Perturbations around this are of the form $\Lambda^{2 n-2 \lambda-1} \hat{p}^{2 n}$ and can be assigned an order $d=2(n-\lambda-1)$.

All perturbations around the trivial point are irrelevant. In contrast, the nontrivial fixed point is unstable; there is always at least one relevant perturbation. 


\section{DISCUSSION}

The method outlined here makes it possible to determine the power counting for the short-range interactions in the presence of a known long-range potential. A crucial feature is that the cut-off is applied in the basis of DW's. One should not try to regulate the long-range as well as the short-range potential.

For the example described here, the repulsive $1 / r^{2}$ potential, this leads (appropriately for Prague) to a baroque power counting involving non-integer orders. Nonetheless the terms in the EFT remain in one-to-one correspondance with observables. For the expansion around the nontrivial fixed point these are the terms of a DW (or "modified") effective-range expansion $[10,14]$. If we write the full phase shift as $\delta=\delta_{L}+\tilde{\delta}_{S}$, where $\delta_{L}$ is due to $V_{L}$ alone, then this expansion has the form

$$
p^{2 \lambda+1}\left[\cot \tilde{\delta}_{S}-\cot \pi\left(\lambda+\frac{1}{2}\right)\right]=\sum_{n=0}^{\infty} C_{2 n} \hat{p}^{2 n},
$$

where $C_{2 n}$ is the coefficient of the perturbation of order $2(n-\lambda-1)$ in the potential.

We have also applied this method to the Coulomb potential, elucidating the power counting for the results of Kong and Ravndal [11], and the attractive $1 / r^{2}$ potential [12]. In the latter case, one has to make the scattering for $V_{L}$ alone well-defined before the method can be applied. This is done by choosing a self-adjoint extension [15] or, more physically, by including a short-range potential as part of $V_{L}$. The need for such an interaction has also been found in the corresponding three-body problems [16].

\section{ACKNOWLEDGMENTS}

This work was supported by the EPSRC. MCB is grateful for the hospitality of the Institute for Nuclear Theory, Seattle, where this work was started.

\section{REFERENCES}

1. S. R. Beane, P. F. Bedaque, W. C. Haxton, D. R. Phillips and M. J. Savage, nucl-th/0008064.

2. S. Weinberg, Physica 96A, 327 (1979).

3. S. Weinberg, Nucl. Phys. B363, 3 (1991).

4. P. F. Bedaque and U. van Kolck, Phys. Lett. B428, 221 (1998).

5. U. van Kolck, Nucl. Phys. A645, 273 (1999).

6. D. B. Kaplan, M. J. Savage, and M. B. Wise, Nucl. Phys. B534, 329 (1998).

7. J. Gegelia, J. Phys. G: Nucl. Part. Phys. 25, 1681 (1999).

8. M. C. Birse, J. A. McGovern and K. G. Richardson, Phys. Lett. B464, 169 (1999).

9. K. G. Wilson and J. G. Kogut, Phys. Rep. 12, 75 (1974).

10. J. M. Blatt and J. D. Jackson, Phys. Rev. 76, 18 (1949); H. A. Bethe, Phys. Rev. 76, 38 (1949).

11. X. Kong and F. Ravndal, Nucl. Phys. A665, 137 (2000).

12. T. Barford and M. C. Birse, in preparation.

13. V. N. Efimov, Sov. J. Nucl. Phys. 12, 589 (1971).

14. H. van Haeringen and L. P. Kok, Phys. Rev. A26, 1218 (1982).

15. A. M. Perelomov and V. S. Popov, Theor. Math. Phys. 4, 664 (1970).

16. P. F. Bedaque, H.-W. Hammer and U. van Kolck, Nucl. Phys. A646, 444 (1999); A676, 357 (2000). 